\title{
Geometric fit of a point set by generalized circles
}

\author{
Mark-Christoph Körner • Jack Brimberg • \\ Henrik Juel · Anita Schöbel
}

Received: 27 April 2010 / Accepted: 18 October 2010 / Published online: 18 November 2010

(C) The Author(s) 2010. This article is published with open access at Springerlink.com

\begin{abstract}
In our paper we approximate a set of given points by a general circle. More precisely, given two norms $k_{1}$ and $k_{2}$ and a set of points in the plane, we consider the problem of locating and scaling the unit circle of norm $k_{1}$ such that the sum of weighted distances between the circumference of the circle and the given points is minimized, where the distance is measured by a norm $k_{2}$. We present results for the general case. In the case that $k_{1}$ and $k_{2}$ are both polyhedral norms, we are able to solve the problem by investigating a finite candidate set.
\end{abstract}

Keywords Circle location $\cdot$ Dimensional facility $\cdot$ Minisum $\cdot$ Polyhedral norms

Mathematics Subject Classification (2000) $\quad$ 62J02 - 65D10 - 90C26 · 90B85 - 97N50

\footnotetext{
M.-C. Körner ( $\square)$

Institute for Numerical and Applied Mathematics, Georg-August-Universität Göttingen, Lotzestr. 16-18, 37083 Göttingen, Germany

e-mail: koerner@math.uni-goettingen.de

J. Brimberg

Royal Military College of Canada, Kingston, ON, Canada

e-mail: jack.brimberg@rmc.ca

J. Brimberg

Groupe d'Études et de Recherche en Analyse des Décisions, Montréal, QC, Canada
}

H. Juel

Technical University of Denmark, Lyngby, Denmark

e-mail: heju@man.dtu.dk

A. Schöbel

Georg-August-Universität Göttingen, Göttingen, Germany

e-mail: schoebel@math.uni-goettingen.de 


\section{Introduction}

Approximating a given point set by a circle, also known as the circle location problem, occurs as a model in different areas. For example Drezner et al. [9] considered the problem of locating a circle on the plane with respect to a point set and suggested it as a model for the out-of-roundness problem. They primarily treated a minimax model, locating the circle so as to minimize the maximum distance between the circle and the point set (see also [3] for further results). In Nievergelt [16] this minimax model is studied in a more general setting and a finite algorithm that computes minimax hyperspheres and hyperplanes is given. The corresponding weighted minisum model to locate a circle so as to minimize the sum of weighted distances between the circle and the point set has been recently investigated in [4] and [17]. Whereas in [4] the planar case is discussed, [17] provides results for higher dimensions, i.e. results for locating minisum hyperspheres. Chan [6] and more recently Chernov and Sapirstein [7] suggested circle location models as estimators in statistical models. Chernov and Sapirstein used the model to estimate the diameter of ancient sherds which were collected during archaeological field work. Karimäki [12] considered a least squares circle location model in order to estimate trajectories of particles. Other applications of circle location models include the design of circular public transportation networks (PTN for short). Circular PTNs are common in practice, e.g., in London, Moscow, Berlin, Hamburg, and Tokyo, circular underground or suburban railways can be found. Circle location models are suited to determine a rough route of a new circular PTN that minimizes the distance from the customers to the PTN. In a subsequent detailed planning, this tentative route can be adapted to local realities (e.g. buildings, watercourses, parks, and nature protection areas). Similarly, ring roads may also be of practical interest; see Pearce [18] and Suzuki [25].

In virtually all papers on circle location problems only the Euclidean case has been studied. Recently a more general case has been considered where circles are defined w.r.t. an arbitrary norm $k$ and distances between points and the circumference of the circle are also measured by the metric induced by the norm $k$, see [2]. In this paper, we generalize the Euclidean view in two respects: First, we locate a convex, symmetric set (i.e. the unit circle with respect to an arbitrary norm $k_{1}$ ) instead of the unit circle with respect to the Euclidean norm $\ell_{2}$. Second, we measure the distance from points to the circumference of the circle in a (maybe different) norm $k_{2}$ instead of using the Euclidean distance. The new formulation would be relevant in cases where distance is measured differently, for example, a PTN where travel distance is rectangular $\left(k_{2}=\ell_{1}\right)$ while the PTN itself has to be circular $\left(k_{1}=\ell_{2}\right)$, rectangular (weighted $l_{\infty}$ ), or any other convex symmetric shape.

Other popular distance functions used to estimate travel distances include the weighted $\ell_{p}$ norm and general polyhedral (or block) norms. Note that the $\ell_{p}$ norm given by

$$
\ell_{p}(X)=\left(\left|x_{1}\right|^{p}+\left|x_{2}\right|^{p}\right)^{\frac{1}{p}}, \quad p \geq 1,
$$

where $X=\left(x_{1}, x_{2}\right) \in \mathbb{R}^{2}$, is a generalization of the rectangular norm ( $\left.p=1\right)$, Euclidean norm $(p=2)$, and Tchebycheff norm $(p \rightarrow \infty)$. There is a large body of literature on the estimation of travel distance by empirical distance functions; see for example the references listed in [5]. Also, the interested reader is referred to classical texts on continuous location models such as $[15,11,8]$.

A large part of this paper is devoted to the case where $k_{1}$ and $k_{2}$ are polyhedral (or block) norms. Polyhedral norms have been used extensively in location theory. For example, Ward and Wendel [26] show that the weighted one-infinity norm (a linear combination of the $\ell_{1}$ and $\ell_{\infty}$ norms) provides a comparable estimator of travel distances as the weighted $\ell_{p}$ norm. 
In addition, polyhedral norms often allow continuous location problems to be reduced to a finite dominating set of candidate solutions that can be resolved by linear programming or related techniques (e.g., see [26,27]). The relative abilities of polyhedral norms and round norms as distance estimators has been a subject of animated discussion for some time (e.g., see [5] and the list of references therein), even though they are equivalent in a limiting sense; that is, by increasing the number of fundamental directions, a polyhedral norm can become as accurate a representation of a round norm as desired. This shows the importance of polyhedral norms for all kinds of applications and that the restriction of $k_{1}$ and $k_{2}$ to the general class of polyhedral norms is still useful in a practical setting.

Related to our problem is the location of a circle on a sphere. This problem is examined in Brimberg et al. [1], and applications in diverse areas, including medical/biological and search-and-rescue, are noted. A discrete formulation of our problem is studied in Labbé et al. [14].

The remainder of the paper is structured as follows. In the next section the main notation is specified, and the general minisum circle location problem is defined. Sections 3 and 4 examine properties of point-circle distances and optimal solutions in the framework of the general problem presented. Section 5 examines the case where only polyhedral norms are used. Polyhedral (or block) norms are often used as piecewise linear approximations of more conventional norms such as Euclidean. Here we show that a finite dominating set exists that provides the basis of a solution algorithm. Finally, Sect. 6 presents our conclusions and suggestions for future research.

\section{Problem statement and notation}

Consider a set of $n \geq 2$ given points (also called fixed points), $A_{j}=\left(a_{j}, b_{j}\right)$ with associated positive weights $w_{j}$, for $j=1, \ldots, n$. Our goal is to find a (general) circle such that the weighted sum of shortest distances from the fixed points to the circumference of the circle is minimized.

To describe our problem, let two norms $k_{1}, k_{2}$ be given. The general circle we want to locate is the scaled unit circle with respect to norm $k_{1}$. It is determined by its center, $X=(x, y)$, and its radius, $r$,

$$
C(X, r)=\left\{Y \in \mathbb{R}^{2}: k_{1}(X-Y)=r\right\} .
$$

Although this definition describes the circumference of a circle, we will be referring to $C(X, r)$ as circle in the following. The distance between a circle $C=C(X, r)$ and a point $A$ is measured through the norm $k_{2}$. It is given as

$$
d(C, A)=\min _{Y \in C} k_{2}(A-Y),
$$

where $Y \in C$ means that $k_{1}(X-Y)=r$. For the fixed points we also use $d_{j}(X, r):=$ $d\left(C, A_{j}\right)$.

The problem may be formulated as

$$
\min f(X, r)=\sum_{j=1}^{n} w_{j} d_{j}(X, r) .
$$

We will refer to this problem as the general minisum circle location problem or (GP) for short. Figures 1, 2, and 3 illustrate different scenarios for the problem using standard 
Fig. 1 Illustration of problem (GP) with Euclidean norm $k_{1}=k_{2}=\ell_{2}$

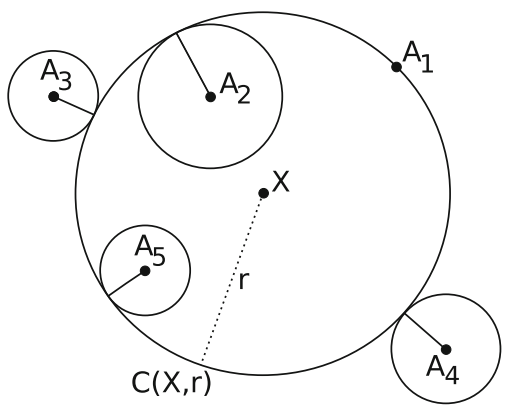

Fig. 2 Illustration of problem (GP) with norms $k_{1}=\ell_{2}$ and $k_{2}=\ell_{1}$

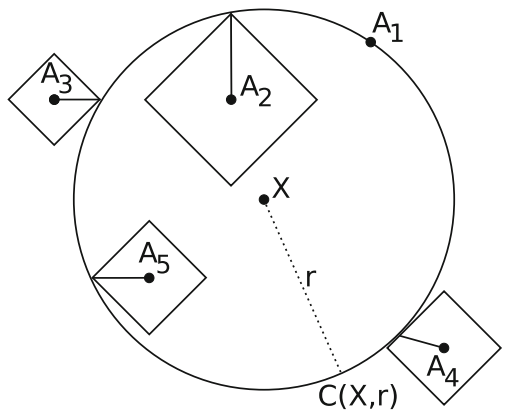

Fig. 3 Illustration of problem (GP) with norms $k_{1}=\ell_{\infty}$ and $k_{2}=\ell_{1}$

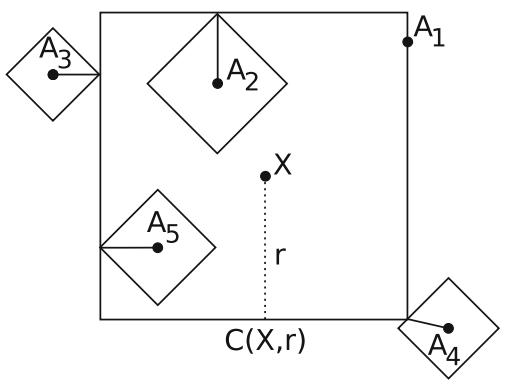

norms such as the Euclidean, rectangular, and Tchebycheff norms. Note that Fig. 1 depicts the original problem with exclusively Euclidean distances studied in e.g. [4, 17], while Figs. 2 and 3 give examples of the new formulation.

Note that the objective function is neither convex nor concave, and may contain several local minima. However, the application of general global optimization techniques may be difficult for the following reasons:

1. The objective function is non-differentiable at points $(X, r) \in \mathbb{R}^{3}$ where the circle $C(X, r)$ intersects one or more fixed points.

2. The subsets of fixed points in the interior and exterior of the circle $C(X, r)$ change depending on $X$ and $r$. This changes the form of the objective function.

3. Limiting solutions $(r \rightarrow \infty)$ are possible; hence, the search space is unbounded.

As a result of Point 2, dc-programming is not applicable. As a result of point 3, classical branch-and-bound methods such as big square small square (BSSS), generalized big square small square (GBSSS), or big triangle small triangle (BTST) (see e.g. [20,4,24,10,22]) that are used extensively to solve non-convex location problems, are also not applicable. On the 
other hand, constrained problems, for example, where the radius is fixed or bounded, are amenable to BSSS (see e.g. [4,24,21]).

Summarizing, fitting a point set by a circle is a challenging problem which asks for a new methodology since the classical approaches of global optimization are not suitable.

\section{Point-circle distance}

In this section we study the point-circle distance $d(C, A)$. First we note that $d(C, A)$ has a nice symmetry property: the distance between the circle $C=C(X, r)$ and the point $A$ coincides with the distance between the point $X$ and the circle $C(A, r)$. This result is shown by the following lemma.

Lemma 1 Let $A, X \in \mathbb{R}^{2}$ and $r \geq 0$. Then, $d(C(X, r), A)=d(C(A, r), X)$.

Proof Let $C_{1}=C(X, r)$ and $C_{2}=C(A, r)$. Let $Y_{1} \in C_{1}$ such that $d\left(C_{1}, A\right)=k_{2}\left(Y_{1}-A\right)$. Let $Y_{2}:=A+X-Y_{1}$. Note that $Y_{2} \in C_{2}$, since $k_{1}\left(Y_{2}-A\right)=k_{1}\left(X-Y_{1}\right)=r$. We have

$$
k_{2}\left(Y_{2}-X\right)=k_{2}\left(A+X-Y_{1}-X\right)=k_{2}\left(A-Y_{1}\right)=d\left(C_{1}, A\right),
$$

hence $d\left(C_{2}, X\right) \leq d\left(C_{1}, A\right)$. Now we choose $Z_{1} \in C_{2}$ such that $d\left(C_{2}, X\right)=k_{2}\left(Z_{1}-\right.$ $X)$. Analogous to the previous case we obtain $d\left(C_{2}, X\right) \geq d\left(C_{1}, A\right)$; that is, $d\left(C_{1}, A\right)=$ $d\left(C_{2}, X\right)$.

It can be shown that the point-circle distance $d(C(X, r), A)$ behaves on certain regions like a concave and convex function, respectively.

Lemma 2 The point-circle distance $d(C(X, r), A)$ between a circle $C(X, r)$ and a fixed point $A$ is

(i) concave in $(X, r)$ on the set

$$
V_{A}:=\left\{(X, r) \in \mathbb{R}^{2} \times\left[0, \infty\left[: k_{1}(X-A) \leq r\right\},\right.\right.
$$

and

(ii) convex in $(X, r)$ on any convex set $U \subseteq\left(\mathbb{R}^{2} \times\left[0, \infty[) \backslash V_{A}\right.\right.$.

Proof Convexity. Let $U \subseteq\left(\mathbb{R}^{2} \times\left[0, \infty[) \backslash V_{A}\right.\right.$ be a convex set. Consider any two points $\left(X_{1}, r_{1}\right),\left(X_{2}, r_{2}\right) \in U$. Let $X_{3}:=\lambda X_{1}+(1-\lambda) X_{2}$ and $r_{3}:=\lambda r_{1}+(1-\lambda) r_{2}$ for some $\lambda \in[0,1]$. Using Lemma 1 , we get

$$
d\left(C\left(X_{i}, r_{i}\right), A\right)=d\left(C\left(A, r_{i}\right), X_{i}\right)=k_{2}\left(X_{i}-Z_{i}\right)
$$

where $Z_{i} \in C\left(A, r_{i}\right)$ minimizes the $k_{2}$-distance from $X_{i}$ to $C\left(A, r_{i}\right), i=1,2,3$. We have

$$
k_{1}\left(\lambda Z_{1}+(1-\lambda) Z_{2}-A\right) \leq \lambda k_{1}\left(Z_{1}-A\right)+(1-\lambda) k_{1}\left(Z_{2}-A\right)=r_{3}
$$

that is, we may conclude that $\lambda Z_{1}+(1-\lambda) Z_{2}$ is either on $C\left(A, r_{3}\right)$ or in its interior. Since $X_{3}$ does not belong to $C\left(A, r_{3}\right)$ or its interior we obtain

$$
\begin{aligned}
k_{2}\left(X_{3}-Z_{3}\right) & =\min \left\{k_{2}\left(X_{3}-Z\right): Z \in C\left(A, r_{3}\right)\right\} \\
& =\min \left\{k_{2}\left(X_{3}-Z\right): k_{1}(Z-A) \leq r_{3}\right\} \\
& \leq k_{2}\left(X_{3}-\left(\lambda Z_{1}+(1-\lambda) Z_{2}\right)\right) \\
& =k_{2}\left(\lambda X_{1}+(1-\lambda) X_{2}-\left(\lambda Z_{1}+(1-\lambda) Z_{2}\right)\right) .
\end{aligned}
$$


Thus

$$
\begin{aligned}
d\left(C\left(X_{3}, r_{3}\right), A\right) & =d\left(C\left(A, r_{3}\right), X_{3}\right) \\
& =k_{2}\left(X_{3}-Z_{3}\right) \\
& \leq k_{2}\left(\lambda\left(X_{1}-Z_{1}\right)+(1-\lambda)\left(X_{2}-Z_{2}\right)\right) \\
& \leq \lambda k_{2}\left(X_{1}-Z_{1}\right)+(1-\lambda) k_{2}\left(X_{2}-Z_{2}\right) \\
& =\lambda d\left(C\left(A, r_{1}\right), X_{1}\right)+(1-\lambda) d\left(C\left(A, r_{2}\right), X_{2}\right) \\
& =\lambda d\left(C\left(X_{1}, r_{1}\right), A\right)+(1-\lambda) d\left(C\left(X_{2}, r_{2}\right), A\right) .
\end{aligned}
$$

Concavity. Let $\partial V_{A}:=V_{A} \backslash \operatorname{int}\left(V_{A}\right)$ denote the boundary of $V_{A}$. For any point $(X, r) \in$ $\partial V_{A}, r>0$, let $H(X, r)$ denote a supporting plane of $V_{A}$ at $(X, r) . H(X, r)$ exists due to the convexity of the cone $V_{A}$, and contains the ray from apex $(A, 0)$ passing through $(X, r)$. (Note: if $k_{1}$ is a smooth norm then $H(X, r)$ is uniquely defined. If $k_{1}$ has corners then infinitely many supporting planes will occur at each corner, in which case anyone may be selected arbitrarily.) Furthermore, we define

$$
\mathscr{H}:=\left\{H(X, r):(X, r) \in \partial V_{A} \backslash(A, 0)\right\} .
$$

For any plane $H \in \mathscr{H}$ and any point $(X, r) \in V_{A}$ we define the distance

$$
d(X, r, H):=\min \left\{k_{2}(X-Y):(Y, r) \in H\right\} .
$$

Now we consider an arbitrary point $(X, r) \in V_{A}$. Let $Y \in C(A, r)$ such that

$$
k_{2}(X-Y)=d(C(A, r), X)=d(C(X, r), A) .
$$

Due to the definition of $\mathscr{H}$ we have

$$
d(C(X, r), A)=k_{2}(X-Y) \leq d(X, r, H), \forall H \in \mathscr{H} .
$$

Furthermore, specifically for $H(Y, r)$ we have $(Y, r) \in H$, and therefore,

$$
d(C(X, r), A)=k_{2}(X-Y)=d(X, r, H) .
$$

It follows that

$$
d(C(X, r), A)=\min \{d(X, r, H): H \in \mathscr{H}\}, \forall(X, r) \in V_{A} .
$$

But the distance $d(X, r, H)$ between $(X, r)$ and a supporting plane $H$ is a linear function of $(X, r)$ in $V_{A}$, as we show in the lemma following this proof. Thus, $d(C(X, r), A)$ is the point-wise minimum of a (infinite) set of linear functions, and is itself concave, for all $(X, r) \in V_{A}$.

Lemma 3 Let $A$ be a fixed point and $H \subseteq \mathbb{R}^{3}$ be a supporting plane of the set $V_{A}$ (see Lemma 2 for the definition of $V_{A}$ ). The distance

$$
d(X, r, H)=\min \left\{k_{2}(X-Y):(Y, r) \in H\right\},
$$

is a linear function of $(X, r)$ in $V_{A}$.

Proof Let us assume that $\alpha \in \mathbb{R}^{3}, \alpha \neq 0$, and $\beta \in \mathbb{R}$ such that

$$
H=\left\{\left(\begin{array}{l}
y_{1} \\
y_{2} \\
y_{3}
\end{array}\right) \in \mathbb{R}^{3}: \alpha_{1} y_{1}+\alpha_{2} y_{2}+\alpha_{3} y_{3}=\beta\right\} .
$$


The set

$$
H(r):=\left\{Y \in \mathbb{R}^{2}:(Y, r)^{t} \in H\right\}=\left\{\left(\begin{array}{l}
y_{1} \\
y_{2}
\end{array}\right) \in \mathbb{R}^{2}: \alpha_{1} y_{1}+\alpha_{2} y_{2}=\beta-\alpha_{3} r\right\}
$$

is a straight line in $\mathbb{R}^{2}$. Hence,

$$
d(X, r, H)=\min \left\{k_{2}(X-Y): Y \in H(r)\right\}
$$

is the $k_{2}$-distance between the point $X \in \mathbb{R}^{2}$ and the straight line $H(r) \subseteq \mathbb{R}^{2}$. For the distance between a point $X=\left(x_{1}, x_{2}\right)^{t}$ and a straight line $H(r)$ we can apply the formula stated in Corollary 1.1 in [19] and obtain

$$
\begin{aligned}
d(X, r, H) & =\min \left\{k_{2}(X-Y): Y \in H(r)\right\} \\
& =\left|\beta-\alpha_{3} r-\alpha_{1} x_{1}-\alpha_{2} x_{2}\right| / k_{2}^{\circ}(\bar{\alpha}),
\end{aligned}
$$

where $\bar{\alpha}:=\left(\alpha_{1}, \alpha_{2}\right)^{t}$ and $k_{2}^{\circ}$ is the dual (or polar) norm of $k_{2}$. Since the denominator $k_{2}^{\circ}(\bar{\alpha})$ does not depend on $(X, r)$ it follows that $d(X, r, H)$ is linear in $(X, r)$ on both half-spaces defined by $H$. With the fact that $V_{A}$ is included in a half-space defined by $H$ we obtain the assertion.

Now we show that the point $A$ is a local maximum of $h_{r}(X):=d(C(X, r), A)$. Note that this result proves that the center $X$ of a circle $C(X, r)$ (defined w.r.t. norm $k_{1}$ ) is a point within $C(X, r)$ which has the greatest $k_{2}$-distance to the circumference of $C(X, r)$. This property may also apply to other points within the circle; that is, the stronger formulation $h_{r}(A)>h_{r}(X)$ is not generally true.

Lemma 4 Let $A \in \mathbb{R}^{2}$ be a given point, $r>0$ and $h_{r}(X)=d(C(X, r), A)$. Then $h_{r}(A) \geq$ $h_{r}(X)$ for all $X \in\left\{Y: k_{1}(Y-A) \leq r\right\}$.

Proof First note that

$$
h_{r}(X)=d(C(A, r), X)=\min _{Y \in C(A, r)} k_{2}(X-Y)
$$

is concave in $X$ on the set $\left\{Y: k_{1}(Y-A) \leq r\right\}$, see Lemmas 1 and 2. Let us assume that there exists $B \in\left\{Y: k_{1}(Y-A) \leq r\right\}$ such that $h_{r}(B)>h_{r}(A)$. Let $P \in C(A, r)$ such that $h_{r}(B)=k_{2}(B-P)$. We define

$$
\begin{aligned}
& B^{\prime}:=A-(B-A), \\
& P^{\prime}:=A-(P-A) .
\end{aligned}
$$

Then, we have $k_{1}\left(A-P^{\prime}\right)=k_{1}(P-A)=r$; that is $P^{\prime} \in C(A, r)$. Furthermore we have $k_{2}\left(B^{\prime}-P^{\prime}\right)=k_{2}(B-P)$. We obtain

$$
h_{r}\left(B^{\prime}\right) \leq k_{2}\left(B^{\prime}-P^{\prime}\right)=k_{2}(B-P)=h_{r}(B) .
$$

Now, let $Q^{\prime} \in C(A, r)$ such that $h_{r}\left(B^{\prime}\right)=k_{2}\left(B^{\prime}-Q^{\prime}\right)$ and define $Q:=A-\left(Q^{\prime}-A\right)$. Analogously to the previous case we obtain $h_{r}(B) \leq h_{r}\left(B^{\prime}\right)$, hence $h_{r}(B)=h_{r}\left(B^{\prime}\right)$. Since $A=\frac{1}{2}\left(B+B^{\prime}\right)$ we end up in a contradiction to the concavity of $h_{r}$ :

$$
h_{r}\left(\frac{1}{2}\left(B+B^{\prime}\right)\right)=h_{r}(A)<h_{r}(B)=\frac{1}{2} h_{r}(B)+\frac{1}{2} h_{r}\left(B^{\prime}\right) .
$$


For the equal norms case $k_{1}=k_{2}=: k$ a simple formula for the point-circle distance between a point $A$ and a circle $C=C(X, r)$ is known:

$$
d(C, A)=|k(X-A)-r|,
$$

see [2]. For the general case $k_{1} \neq k_{2}$ this formula does not hold. Let us define

$$
\begin{aligned}
K & :=\max _{X: k_{1}(X)=1} k_{2}(X), \\
G & :=\min _{X: k_{1}(X)=1} k_{2}(X),
\end{aligned}
$$

i.e. for all $A, X \in \mathbb{R}^{2}, A \neq X$, we have

$$
G \leq \frac{k_{2}(A-X)}{k_{1}(A-X)} \leq K .
$$

As an example, for $k_{1}=\ell_{1}, k_{2}=\ell_{2}$ we obtain that $K=1$ and $G=\frac{1}{2} \sqrt{2}$.

We are now able to compute a simple upper bound for the point-circle distance using the distance $k_{1}$.

\section{Lemma 5 Let $A \neq X$. Then}

$$
d(C(X, r), A) \leq \frac{k_{2}(A-X)}{k_{1}(A-X)}\left|k_{1}(A-X)-r\right| \leq K\left|k_{1}(A-X)-r\right| .
$$

Proof Let $\lambda \geq 0$ such that $A^{\prime}:=X+\lambda(A-X) \in C(X, r)$. We obtain

$$
k_{1}\left(X-A^{\prime}\right)=r \Leftrightarrow \lambda k_{1}(A-X)=r \Leftrightarrow \lambda=\frac{r}{k_{1}(A-X)} .
$$

With this fact we get

$$
\begin{aligned}
d(C(X, r), A) \leq k_{2}\left(A-A^{\prime}\right) & =k_{2}(A-X-\lambda(A-X))=|1-\lambda| k_{2}(A-X) \\
& =\left|1-\frac{r}{k_{1}(A-X)}\right| k_{2}(A-X)=\frac{k_{2}(A-X)}{k_{1}(A-X)}\left|k_{1}(A-X)-r\right| .
\end{aligned}
$$

We can also use $k_{2}$ to get the following lower bound on the point-circle distance.

\section{Lemma 6}

- If $k_{1}(A-X) \geq r$ we have $d(C(X, r), A) \geq k_{2}(A-X)-K r$,

- if $k_{1}(A-X) \leq r$ we have $d(C(X, r), A) \geq G r-k_{2}(A-X)$.

Proof Let $A^{\prime} \in C(X, r)$ such that $d(C(X, r), A)=k_{2}\left(A-A^{\prime}\right)$.

1. Let $k_{1}(A-X) \geq r$. Then

$$
\begin{aligned}
k_{2}(A-X) & \leq k_{2}\left(A-A^{\prime}\right)+k_{2}\left(A^{\prime}-X\right)=d(C(X, r), A)+k_{2}\left(A^{\prime}-X\right) \\
& \leq d(C(X, r), A)+K k_{1}\left(A^{\prime}-X\right)=d(C(X, r), A)+K r .
\end{aligned}
$$

2. Let $k_{1}(A-X) \leq r$. Then, we have

$$
k_{2}\left(A^{\prime}-X\right) \leq k_{2}\left(A-A^{\prime}\right)+k_{2}(A-X)=d(C(X, r), A)+k_{2}(A-X),
$$

hence we obtain

$$
\begin{aligned}
d(C(X, r), A) & \geq k_{2}\left(A^{\prime}-X\right)-k_{2}(A-X) \\
& \geq G k_{1}\left(A^{\prime}-X\right)-k_{2}(A-X)=G r-k_{2}(A-X)
\end{aligned}
$$


Fig. 4 Illustration of Example 1 with fixed points $A_{1}, \ldots, A_{5}$ and an optimal circle $C((8,8), 8)$

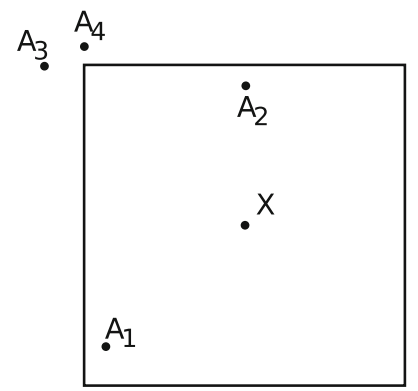

\section{Properties of optimal solutions}

In this section we study properties of optimal solutions for (GP). We also discuss properties that are known for the Euclidean case $k_{1}=k_{2}=\ell_{2}$, see [4], and the equal norms case $k_{1}=k_{2}$, see [2], but do not apply to general minisum circle location problems.

We start with the main result for locating a median circle with Euclidean distances (i.e. $k_{1}=k_{2}=\ell_{2}$ ). The following incidence property is known here: an optimal circle must intersect at least two of the fixed points (see [4]). This property is also known for Euclidean line location problems ([28]) where it has been generalized to all distances derived from norms ([23]). Unfortunately it is not possible to extend this incidence property also to the case of locating a general circle. Even worse, there exist cases in which no optimal circle intersects any of the fixed points. Therefore, also the weak incidence property (at least one optimal solution intersects one fixed point) known for the equal norms case, i.e. if $k_{1}=k_{2}$, does not not apply to (GP).

This negative result is shown by the following example:

Example 1 Let $k_{1}=\ell_{\infty}$ and $k_{2}=\ell_{1}$, and consider

$$
A_{1}=(1,2)^{t}, A_{2}=(8,15)^{t}, A_{3}=(-2,16)^{t}, A_{4}=(0,17)^{t}, A_{5}=(16,20)^{t}
$$

with weights $w_{1}=1.1, w_{2}=2.2, w_{3}=w_{4}=1$, and $w_{5}=1.05$. Using the results of Sect. 5 we obtain that any optimal circle minimizing the weighted sum of distances to the fixed points is contained in the set

$$
O p t:=\left\{C(X, r): X=(8,8)^{t}+(r-8)(1,-1)^{t}, r \geq 8\right\} .
$$

Any optimal circle $C \in O p t$ has an objective function value of

$$
1.1 \cdot 1+2.2 \cdot 1+2+1+1.05 \cdot 4=10.5 \text {. }
$$

In particular, none of the optimal circles intersect any of the existing points, see also Fig. 4.

In [2] it is shown that the pseudo-halving property holds for problem (GP) whenever $k_{1}=k_{2}$, i.e., any optimal circle for the equal norm case must satisfy

$$
\left|\sum_{j \in J_{-}} w_{j}-\sum_{j \in J_{+}} w_{j}\right| \leq \sum_{j \in J_{0}} w_{j},
$$

where $J_{-}:=\left\{j \in\{1, \ldots, n\}: k\left(X-A_{j}\right)<r\right\}, J_{+}:=\left\{j \in\{1, \ldots, n\}: k\left(X-A_{j}\right)>r\right\}$, and $J_{0}:=\left\{j \in\{1, \ldots, n\}: k\left(X-A_{j}\right)=r\right\}$. This property is also known for the median 
line location problem, see [23]. But Example 1 also shows that the pseudo-halving property does not hold for problem (GP) in general, since the optimal circle $C((8,8), 8)$ does not satisfy (1).

However, there are some interesting properties for the equal norm case $k_{1}=k_{2}$ which may be extended to the general case. One of these is that for (GP) a degenerated circle with radius $r=0$ cannot be an optimal solution.

\section{Lemma 7 The optimal solution of Problem (GP) must have a positive radius.}

Proof The result can be shown by contradiction in a similar fashion as the analog result in [2]. First, we assume that a circle $C^{*}=C\left(X^{*}, r^{*}\right)$ with radius $r^{*}=0$ is a degenerated optimal solution. Since $n \geq 2$, we can then find a fixed point, say $A_{1}$, such that $X^{*} \neq A_{1}$. It follows that $k_{2}\left(X^{*}-A_{1}\right)>0$. Let $C^{\prime}=C\left(X^{\prime}, r^{\prime}\right)$ be any circle intersecting $X^{*}$ and $A_{1}$. Since $C^{*} \subseteq C^{\prime}, d\left(C^{*}, A_{i}\right) \geq d\left(C^{\prime}, A_{i}\right)$ for all $i=1, \ldots, n$. In particular for $i=1$ we obtain a strict inequality, namely

$$
d\left(C^{*}, A_{1}\right)=k_{2}\left(X^{*}-A_{1}\right)>0=k_{2}\left(A_{1}-A_{1}\right)=d\left(C^{\prime}, A_{1}\right) .
$$

Together

$$
f\left(C^{*}\right)=\sum_{i=1}^{n} w_{i} d\left(C^{*}, A_{i}\right)>\sum_{i=1}^{n} w_{i} d\left(C^{\prime}, A_{i}\right)=f\left(C^{\prime}\right) .
$$

Thus, $C^{*}$ cannot be optimal.

From Lemma 7 it follows that a point can never be an optimal solution of the general circle location problem (GP) with more than one fixed point. However, another extreme is possible. The general minisum circle location problem is unbounded in the sense that increasing the radius may continuously improve the objective value; that is, it is possible that a minimizer for (GP) does not exist. In this case a straight line (the local limit of a smooth circle with $r \rightarrow \infty$ ) or a bent line (the local limit of a circle with corner points) may solve Problem (GP). For the Euclidean case $k_{1}=k_{2}=\ell_{2}$ an example where a straight line is the optimal limiting solution is given in [4]. However, for this case mild conditions exist guaranteeing that optimal solutions always have a finite radius; only general positions of the fixed points and $n \geq 5$ is required, see [4]. In [2] this result was generalized to norms $k_{1}=k_{2}$ with ellipsoids as unit circles. For (GP) analog results seem to be not possible as the following example shows.

Example 2 Let $k_{1}$ be the Euclidean norm; that is, $k_{1}=\ell_{2}$. Furthermore, let $k_{2}$ be a norm having a "long and thin" ellipsoid as unit circle which is rotated counterclockwise by $45^{\circ}$. Consider the fixed points depicted in Fig. 5 and assume that sufficiently large weights are associated to $A_{1}$ and $A_{2}$. For each of the four remaining points assume a weight of 1 . Then, an optimal straight line to the corresponding linear facility problem is the line $\ell_{12}$ depicted in Fig. 5. Furthermore, the large weights associated to $A_{1}$ and $A_{2}$ ensures that any optimal circle passes through to $A_{1}$ and $A_{2}$. However, Fig. 5 shows that such a circle is always inferior to the straight line $\ell_{12}$ : The lower circle shown in the figure has the same distance to $A_{3}$ and $A_{6}$ as does the straight line $\ell_{12}$. The circle is closer to $A_{4}$, however, its distance to $A_{5}$ increases dramatically compared to the distance between $A_{5}$ and $\ell_{12}$ such that the objective function value of the lower circle is greater than the objective function value of $\ell_{12}$. By symmetry the same holds for the upper circle, and hence for all circles passing through $A_{1}$ and $A_{2}$. 


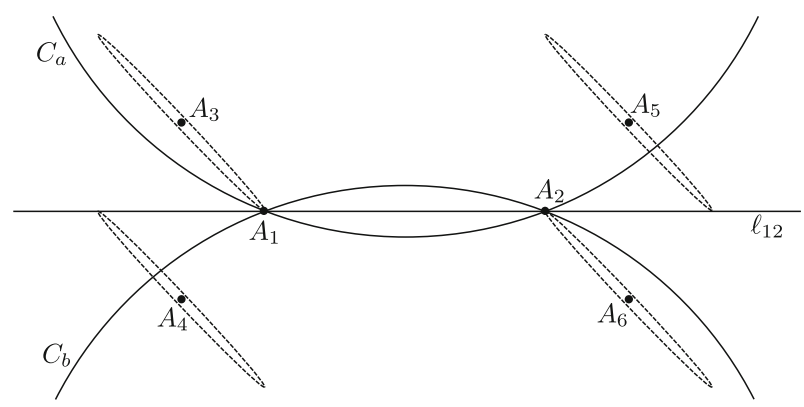

Fig. 5 Illustration of Example 2. Dashed lines are translated unit circles of norm $k_{2}$

We close this section with a dominance criterion for (GP), which is analogous to the majority theorem of Witzgall [29] for location of a point. For this purpose we define

$$
\text { Test }_{j}:=2 w_{j}-\sum_{i=1}^{n} w_{i},
$$

for $j=1, \ldots, n$.

Lemma 8 Suppose an optimal solution of finite radius, $C=C(X, r)$, exists. If Test ${ }_{j}>0$ for some $j \in\{1, \ldots, n\}$, then $C=C(X, r)$ must intersect the fixed point $A_{j}$, i.e., $A_{j} \in C$. If Test ${ }_{j}=0$, then there exists at least one such optimal solution that intersects $A_{j}$.

Proof Let Test $1>0$. Let $C=C(X, r)$ be an optimal solution for (GP) with $r<\infty$ and $A_{1} \notin C$. We show that $A_{1} \notin C$ is a contradiction to the optimality of $C$. To this end we construct a circle $C^{\prime}=C\left(X^{\prime}, r\right)$ such that $f\left(C^{\prime}\right)<f(C)$.

Let $Y_{i} \in C$ such that $k_{2}\left(A_{i}-Y_{i}\right)=d\left(C, A_{i}\right), i=1, \ldots, n$. Furthermore, let $X^{\prime}=$ $X+A_{1}-Y_{1}$ and $C^{\prime}:=C\left(X^{\prime}, r\right)$. Then, we have

$$
k_{1}\left(Y_{i}+A_{1}-Y_{1}-X^{\prime}\right)=k_{1}\left(Y_{i}-X\right)=r
$$

for all $i=1, \ldots, n$; that is, $Y_{i}+A_{1}-Y_{1} \in C^{\prime}$. In particular we obtain $d\left(C^{\prime}, A_{1}\right)=0$ and finally

$$
\begin{aligned}
f\left(C^{\prime}\right) & =\sum_{i=2}^{n} w_{i} d\left(C^{\prime}, A_{i}\right) \\
& \leq \sum_{i=2}^{n} w_{i} k_{2}\left(Y_{i}+A_{1}-Y_{1}-A_{i}\right) \\
& \leq \sum_{i=2}^{n} w_{i} k_{2}\left(Y_{i}-A_{i}\right)+\sum_{i=2}^{n} w_{i} k_{2}\left(A_{1}-Y_{1}\right) \\
& =\sum_{i=2}^{n} w_{i} d\left(C, A_{i}\right)+\sum_{i=2}^{n} w_{i} d\left(C, A_{1}\right) \\
& =f(C)-d\left(C, A_{1}\right)\left(w_{1}-\sum_{i=2}^{n} w_{i}\right)=f(C)-d\left(C, A_{1}\right) \cdot \text { Test }_{1},
\end{aligned}
$$

i.e., $f\left(C^{\prime}\right)<f(C)$ if Test $_{1}>0$. If Test $1=0$, then $f\left(C^{\prime}\right) \leq f(C)$, so that $C$ optimal implies $C^{\prime}$ is also optimal. 


\section{Polyhedral norms}

In this section we analyze the case in which both norms $k_{1}$ and $k_{2}$ are polyhedral norms; that is

$$
\begin{aligned}
& k_{1}(X)=\min \left\{\sum\left|\beta_{g}\right|: \sum \beta_{g} E_{g}=X\right\}, \\
& k_{2}(X)=\min \left\{\sum\left|\beta_{g}\right|: \sum \beta_{g} F_{g}=X\right\},
\end{aligned}
$$

where $E_{g}, g=1, \ldots, s$ and $F_{g}, g=1, \ldots, t$, are, respectively, the fundamental directions of the given polyhedral norms $k_{1}$ and $k_{2}$, and define the vertices (or corner points) of the corresponding unit circles. Throughout this section we will assume that the fundamental directions of $k_{1}$ and $k_{2}$ are numbered in counterclockwise order; in particular this implies that $E_{i}$ and $E_{i+1}$ or rather $F_{i}$ and $F_{i+1}$ are adjacent. Note that for a fixed point $A$ the distance $k_{2}(A-X)$ is linear on each cone given by $A$ and two adjacent fundamental directions of $k_{2}$.

We will now develop a solution method based on a tessellation of the $3 \mathrm{D}$ space $\mathbb{R}^{3}$. The main idea of our approach is simple (see Körner et. al [13]): we identify a finite tessellation $\mathscr{T}$ of $\mathbb{R}^{3}$ into convex and not necessarily bounded polytopes $P_{k} \subseteq \mathbb{R}^{3}, k=1, \ldots, K$, such that the objective function of (GP) is concave on each polytope $P_{k}$. Let $\operatorname{Ext}(\mathscr{T}) \subseteq \mathbb{R}^{3}$ denote the set of vertices of polytopes belonging to $\mathscr{T}$. Then, $\operatorname{Ext}(\mathscr{T})$ is a finite dominating set (FDS for short) for (GP) with polyhedral norms and we can solve (GP) by testing all points in $\operatorname{Ext}(\mathscr{T})$.

In order to obtain the tessellation $\mathscr{T}$ and the FDS $\operatorname{Ext}(\mathscr{T})$, we first define a finite tessellation for each fixed point $A_{i}$ such that the point circle distance $d_{i}(X, r)$ is concave on each cell of the tessellation.

Tessellation for $d_{i}(X, r)$

In order to obtain a tessellation for the point-circle distance $d_{i}(X, r)$ let us fix $A_{i}$ and $(X, r)$. We note that $d_{i}(X, r)$ can be interpreted as the radius of the smallest $k_{2}$-circle with center $A_{i}$ that touches the $k_{1}$-circle $C(X, r)$; that is,

$$
d_{i}(X, r)=\min \left\{v \geq 0: C_{1}(X, r) \cap C_{2}\left(A_{i}, v\right) \neq \emptyset\right\},
$$

where

$$
C_{1}(X, r):=\left\{Y: k_{1}(X-Y)=r\right\} \text { and } C_{2}\left(A_{i}, v\right):=\left\{Y: k_{2}\left(A_{i}-Y\right)=v\right\} .
$$

Since $C_{1}$ and $C_{2}$ are polyhedrons which always intersect at a vertex of one of them, we can distinguish the following cases.

- $C_{2}$ touches $C_{1}$ in a vertex of $C_{1}$. Let $E \in \operatorname{Ext}\left(B_{1}\right)$, where $B_{1}$ is the unit ball of $k_{1}$ $\left(B_{1}=\left\{Y: k_{1}(Y) \leq 1\right\}\right)$, be the fundamental direction of $k_{1}$ that defines this vertex of $C_{1}$. Then, we have

$$
d_{i}(X, r)=k_{2}\left(X+r E-A_{i}\right) .
$$

- $C_{2}$ touches $C_{1}$ in a vertex of $C_{2}$. Let $F \in \operatorname{Ext}\left(B_{2}\right)$, where $B_{2}$ is the unit ball of $k_{2}$ $\left(B_{2}=\left\{Y: k_{2}(Y) \leq 1\right\}\right)$, be the corresponding fundamental direction of $k_{2}$. Then, we have

$$
d_{i}(X, r)=\min \left\{|\lambda|: A_{i}+\lambda F \in C_{1}\right\} .
$$




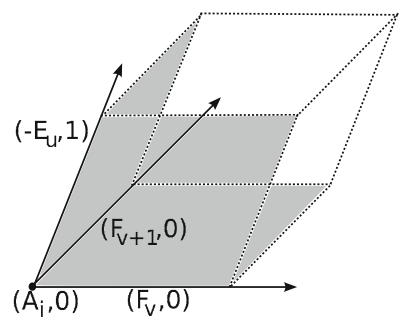

(a) A cone of type $N_{i}(u, v)$

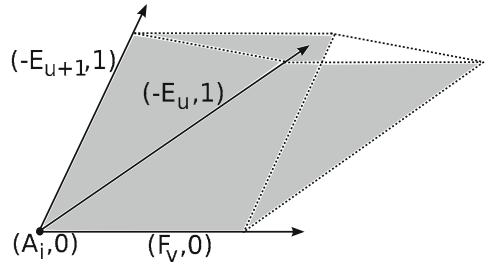

(b) A cone of type $M_{i}(u, v)$

Fig. 6 Example for cones of type $N_{i}(u, v)$ and $M_{i}(u, v)$. Dashed lines: reference lines to point out the dimensional shape of the cone. Facets in dark gray: part of the boundary of the cone

Let $g_{i, j}(X, r):=k_{2}\left(X+r E_{j}-A_{i}\right)$ for any $(X, r) \in \mathbb{R}^{2} \times[0, \infty), i=1, \ldots, n, j=$ $1, \ldots, s$. Furthermore, let $h_{i, j}(X, r):=\min \left\{|\lambda|: A_{i}+\lambda F_{j} \in C_{1}(X, r)\right\}(\min \emptyset:=\infty)$ for any $(X, r) \in \mathbb{R}^{2} \times[0, \infty), i=1, \ldots, n, j=1, \ldots, t$. It follows that

$$
d_{i}(X, r)=\min \left\{\min _{j=1, \ldots, s} g_{i, j}(X, r), \min _{j=1, \ldots, t} h_{i, j}(X, r)\right\} .
$$

Let $g_{i}(X, r):=\min _{j=1, \ldots, s} g_{i, j}(X, r)$ and $h_{i}(X, r):=\min _{j=1, \ldots, t} h_{i, j}(X, r)$. We now identify a cell tessellation of $\mathbb{R}^{3}$ such that $g_{i}$ is concave on each cell and another cell tessellation such that $h_{i}$ is concave on each cell. Then, combining both tessellations we obtain a cell tessellation of $\mathbb{R}^{3}$ such that $d_{i}(X, r)=\min \left\{g_{i}(X, r), h_{i}(X, r)\right\}$ is concave on each cell.

\section{Case 1.}

We start with the function $g_{i}$ and define for any $u=1, \ldots, s, v=1, \ldots, t$, and $i=1, \ldots, n$

$$
N_{i}(u, v):=\left\{\left(\begin{array}{l}
A_{i} \\
0
\end{array}\right)+\alpha_{1}\left(\begin{array}{l}
-E_{u} \\
1
\end{array}\right)+\alpha_{2}\left(\begin{array}{l}
F_{v} \\
0
\end{array}\right)+\alpha_{3}\left(\begin{array}{l}
F_{v+1} \\
0
\end{array}\right): \alpha_{1}, \alpha_{2}, \alpha_{3} \geq 0\right\} .
$$

$N_{i}(u, v)$ is a convex cone in $\mathbb{R}^{3}$ with apex $\left(A_{i}, 0\right)^{t}$. An example for $N_{i}(u, v)$ is depicted in Fig. 6.

Let $\left(X_{1}, r_{1}\right)^{t} \neq\left(X_{2}, r_{2}\right)^{t} \in N_{i}(u, v)$ for some fixed values $u, v$. Then, there exist nonnegative $\alpha_{k 1}, \alpha_{k 2}$, and $\alpha_{k 3}$ such that

$$
\left(\begin{array}{l}
X_{k} \\
r_{k}
\end{array}\right)=\left(\begin{array}{l}
A_{i} \\
0
\end{array}\right)+\alpha_{k 1}\left(\begin{array}{l}
-E_{u} \\
1
\end{array}\right)+\alpha_{k 2}\left(\begin{array}{l}
F_{v} \\
0
\end{array}\right)+\alpha_{k 3}\left(\begin{array}{l}
F_{v+1} \\
0
\end{array}\right),
$$

$k=1,2$. Since $\alpha_{k 1}=r_{k}, k=1,2$, we obtain

$$
\begin{aligned}
g_{i, u}\left(X_{1}+X_{2}-A_{i}, r_{1}+r_{2}\right) & =k_{2}\left(X_{1}+X_{2}+\left(r_{1}+r_{2}\right) E_{u}-2 A_{i}\right) \\
& =k_{2}\left(\left(\alpha_{12}+\alpha_{22}\right) F_{v}+\left(\alpha_{13}+\alpha_{23}\right) F_{v+1}\right) \\
& =\alpha_{12}+\alpha_{22}+\alpha_{13}+\alpha_{23} \\
& =k_{2}\left(\alpha_{12} F_{v}+\alpha_{13} F_{v+1}\right)+k_{2}\left(\alpha_{22} F_{v}+\alpha_{23} F_{v+1}\right) \\
& =k_{2}\left(X_{1}+r_{1} E_{u}-A_{i}\right)+k_{2}\left(X_{2}+r_{2} E_{u}-A_{i}\right) \\
& =g_{i, u}\left(X_{1}, r_{1}\right)+g_{i, u}\left(X_{2}, r_{2}\right) ;
\end{aligned}
$$

that is, $g_{i, u}(X, r)$ is an affine linear function (with translation $\left.\left(A_{i}, 0\right)^{t}\right)$ on each cone $N_{i}(u, v), v=1, \ldots, t$. 
Now, consider the cones $N_{i}(u, v), u=1, \ldots, s, v=1, \ldots, t$. Define a cell as a maximal connected set that is either within or outside these cones; that is, any cell $\mathscr{C}$ is a greatest convex polygon such that $\operatorname{int}(\mathscr{C}) \cap N_{i}(u, v) \in\{\emptyset, \operatorname{int}(\mathscr{C})\}$ for all $u=1, \ldots, s$ and $v=1, \ldots, t$. Let $\mathscr{T}_{i 1}$ be the set that contains all cells we obtain in this way. $\mathscr{T}_{i 1}$ is a tessellation of $\mathbb{R}^{3}$ into a finite set of cells. On each cell $\mathscr{C} \in \mathscr{T}_{i 1}$ each function $g_{i, u}(X, r)$ is affine linear. Therefore, we obtain that $g_{i}(X, r)$ is a concave function on each cell $\mathscr{C} \in \mathscr{T}_{i 1}$.

\section{Case 2.}

We consider the function $h_{i}(X, r):=\min _{j=1, \ldots, t} h_{i, j}(X, r)$. We define for any $u=1, \ldots, s$, $v=1, \ldots, t$, and $i=1, \ldots, n$

$$
h_{i, v}^{u}(X, r):=\min \left\{\lambda \geq 0: A_{i}+\lambda F_{v} \in S_{u}(X, r)\right\},
$$

where $S_{u}(X, r):=\left\{X+\alpha_{1} E_{u}+\alpha_{2} E_{u+1}: \alpha_{1}+\alpha_{2}=r, \alpha_{1}, \alpha_{2} \geq 0\right\}$ and $s+1:=1$. Note that $S_{u}(X, r)$ is a facet of the circle $C_{1}(X, r)$. (If $A_{i}+\lambda F_{v}$ does not intersect $S_{u}(X, r)$, set $h_{i, v}^{u}(X, r)=\infty$.) We obtain

$$
h_{i}(X, r)=\min _{u=1, \ldots, s, v=1, \ldots, t} h_{i, v}^{u}(X, r) .
$$

We now derive a tessellation such that $h_{i, v}^{u}(X, r)$ is linear on any of its cells. To this end we define for any $u=1, \ldots, s, v=1, \ldots, t$, and $i=1, \ldots, n$

$$
M_{i}(u, v):=\left\{\left(\begin{array}{l}
A_{i} \\
0
\end{array}\right)+\alpha_{1}\left(\begin{array}{l}
-E_{u} \\
1
\end{array}\right)+\alpha_{2}\left(\begin{array}{l}
-E_{u+1} \\
1
\end{array}\right)+\alpha_{3}\left(\begin{array}{l}
F_{v} \\
0
\end{array}\right): \alpha_{1}, \alpha_{2}, \alpha_{3} \geq 0\right\} .
$$

$M_{i}(u, v)$ is a convex cone in $\mathbb{R}^{3}$ with apex $\left(A_{i}, 0\right)^{t}$, see Fig. 6 for an illustrative example.

Let $u$ and $v$ be fixed and assume that $\left(-E_{u}, 1\right)^{t},\left(-E_{u+1}, 1\right)^{t}$, and $\left(F_{v}, 0\right)^{t}$ are linearly independent. (Otherwise, $F_{v}$ is parallel to the facet $S_{u}(X, r)$, and thus, $M_{i}(u, v)$ need not be considered.) Consider the cone $M_{i}(u, v)$. Let $(X, r)^{t} \in M_{i}(u, v)$; that is, there exist $\tilde{\alpha}_{1}, \tilde{\alpha}_{2}, \tilde{\alpha}_{3} \geq 0$ such that

$$
\left(\begin{array}{l}
X \\
r
\end{array}\right)=\left(\begin{array}{l}
A_{i} \\
0
\end{array}\right)+\tilde{\alpha}_{1}\left(\begin{array}{l}
-E_{u} \\
1
\end{array}\right)+\tilde{\alpha}_{2}\left(\begin{array}{l}
-E_{u+1} \\
1
\end{array}\right)+\tilde{\alpha}_{3}\left(\begin{array}{l}
F_{v} \\
0
\end{array}\right) .
$$

We show $h_{i, v}^{u}(X, r)=\tilde{\alpha}_{3}$ for all $(X, r) \in M_{i}(u, v)$. To this end note that $A_{i}+\lambda F_{v} \in S_{u}(X, r)$ if and only if

$$
X-\lambda F_{v} \in \bar{S}_{u}\left(A_{i}, r\right):=\left\{A_{i}-\alpha_{1} E_{u}-\alpha_{2} E_{u+1}: \alpha_{1}+\alpha_{2}=r, \alpha_{1}, \alpha_{2} \geq 0\right\} .
$$

Therefore, we obtain

$$
h_{i, v}^{u}(X, r)=\min \left\{\lambda \geq 0: X-\lambda F_{v} \in \bar{S}_{u}\left(A_{i}, r\right)\right\} .
$$

Since $A_{i}-\tilde{\alpha}_{1} E_{u}-\tilde{\alpha}_{2} E_{u+1} \in \bar{S}_{u}\left(A_{i}, r\right)$ we conclude $X-\tilde{\alpha}_{3} F_{v} \in \bar{S}_{u}\left(A_{i}, r\right)$ and hence $h_{i, v}^{u}(X, r) \leq \tilde{\alpha}_{3}$. Due to the linear independence of

$$
\left(-E_{u}, 1\right)^{t},\left(-E_{u+1}, 1\right)^{t},\left(F_{v}, 0\right)^{t}
$$

the representation of $(X, r)^{t}$ given in (2) is unique. Therefore, we may conclude

$$
h_{i, v}^{u}(X, r)=\tilde{\alpha}_{3}
$$

and hence we obtain the following result. 
Fig. 7 Direction lines of norm $k_{2}=\ell_{1}$ through $X_{1}$ and $X_{2}$; circle $C\left(A_{i}, r\right)$

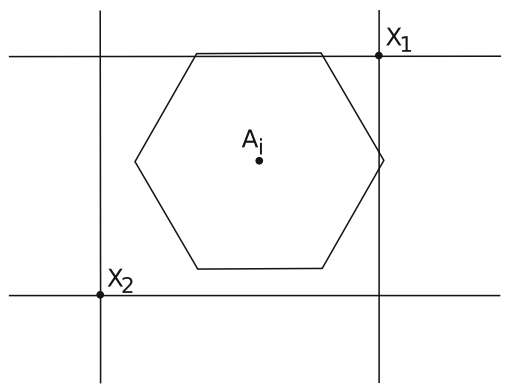

Lemma 9 Let $(u, v) \in\{1, \ldots, s\} \times\{1, \ldots, t\}$ such that

$$
\left(F_{v}, 0\right)^{t},\left(-E_{u}, 1\right)^{t}, \text { and }\left(-E_{u+1}, 1\right)^{t}
$$

are linearly independent. Then, the function $h_{i, v}^{u}(X, r)$ is linear on the cone $M_{i}(u, v)$.

Now, we define a tessellation $\mathscr{T}_{i 2}$ analogously to the tessellation $\mathscr{T}_{i 1}$; that is, we define $\mathscr{T}_{i 2}$ to be the tessellation induced by the cones $M_{i}(u, v), u=1, \ldots, s, v=1, \ldots, t$. Let $\mathscr{C} \in \mathscr{T}_{i 2}$ be a cell and define the set

$$
J_{1}:=\left\{(u, v) \in\{1, \ldots, s\} \times\{1, \ldots, t\}: \mathscr{C} \cap \operatorname{int}\left(M_{i}(u, v)\right)=\emptyset\right\} .
$$

Furthermore, we define $J_{2}:=\{1, \ldots, s\} \times\{1, \ldots, t\} \backslash J_{1}$. We obtain $h_{i, v}^{u}(X, r)=\infty$ for all $(X, r)^{t} \in \mathscr{C}$ if and only if $(u, v) \in J_{1}$. Let $(X, r)^{t} \in \operatorname{int}(\mathscr{C})$. We draw the circle $C\left(A_{i}, r\right)$ and the point $X$. Furthermore we draw the direction lines of $k_{2}$ through $X$, see Fig. 7. Two cases are possible:

- There exists a fundamental direction $F$ of norm $k_{2}$ such that the ray

$$
\{X-\lambda F: \lambda \geq 0\}
$$

intersects $C\left(A_{i}, r\right)$. In this case $J_{2} \neq \varnothing$ and we obtain $h_{i}(X, r)=\min _{(u, v) \in J_{2}} h_{i, v}^{u}$. Since $(X, r)^{t} \in \operatorname{int}(\mathscr{C})$ the vectors

$$
\left(-E_{u}, 1\right)^{t},\left(-E_{u+1}, 1\right)^{t},\left(F_{v}, 0\right)^{t}
$$

must be linearly independent for all $(u, v) \in J_{2}$. Hence for all $(u, v) \in J_{2} h_{i, v}^{u}$ is a linear function, and hence, we obtain that $h_{i}(X, r)$ is concave on $\mathscr{C}$.

- For all fundamental directions $F$ of norm $k_{2}$ the intersection

$$
\{X-\lambda F: \lambda \geq 0\} \cap C\left(A_{i}, r\right)
$$

is empty; that is, $J_{2}=\emptyset$ and hence we obtain $h_{i}(X, r)=\infty$ for all points $(X, r)^{t} \in \mathscr{C}$.

Thus, on any cell of the tessellation $\mathscr{T}_{i 2}, h_{i}(X, r)$ is either concave or it has the value $+\infty$. From Case $1\left(g_{i}(X, r)\right)$ and Case $2\left(h_{i}(X, r)\right)$ we obtain the following result.

Theorem 1 Let $i \in\{1, \ldots, n\}$ and let $\mathscr{T}_{i}$ be the tessellation of $\mathbb{R}^{3}$ we obtain by combining $\mathscr{T}_{i 1}$ and $\mathscr{T}_{i 2}$. Then, the point-circle distance $d_{i}(X, r)$ is concave on each cell $\mathscr{C} \in \mathscr{T}_{i}$. 
Tessellation for (GP)

With Theorem 1 it is straightforward to obtain a tessellation for (GP). We just define $\mathscr{T}$ to be the tessellation we obtain by combining the tessellations $\mathscr{T}_{i}$ for $i=1, \ldots, n$. Then, the point-circle distance $d_{i}(X, r)$ is concave for all $i=1, \ldots, n$ on any cell $\mathscr{C} \in \mathscr{T}$. Thus, also the objective function $f(X, r)=\sum_{i=1}^{n} w_{i} d_{i}(X, r)$ of (GP) is concave on any cell $\mathscr{C} \in \mathscr{T}$, since $w_{i} \geq 0$ for all $i=1, \ldots, n$. Therefore, we obtain the following main result of this section which leads directly to a polynomial algorithm to solve (GP) when $k_{1}$ and $k_{2}$ are both polyhedral norms.

Theorem 2 Let $\operatorname{Ext}(\mathscr{T})$ denote the set that contains all vertices of cells $\mathscr{C} \in \mathscr{T}$. $\operatorname{Ext}(\mathscr{T})$ is a finite dominating set for $(G P)$.

Proof A concave function defined on a polyhedral cell $\mathscr{C}$ attains its minimum in a vertex of $\mathscr{C}$.

Lemma $10 \operatorname{Ext}(\mathscr{T})$ contains at most $\mathscr{O}\left((n s t)^{3}\right)$ points.

Proof Each cone $N_{i}(u, v), M_{i}(u, v)$ has exactly three facets. Any point in $\operatorname{Ext}(\mathscr{T})$ is the intersection of three different facets. There are at most $\mathscr{O}(n s t)$ different facets. Hence, we have at most $\mathscr{O}\left((n s t)^{3}\right)$ points in $\operatorname{Ext}(\mathscr{T})$.

The FDS $\operatorname{Ext}(\mathscr{T})$ gives rise to a simple solution method to (GP) with polyhedral norms $k_{1}$ and $k_{2}$ :

1. Compute all points $(X, r) \in \operatorname{Ext}(\mathscr{T})$.

2. For all $(X, r) \in \operatorname{Ext}(\mathscr{T})$ with $r>0$, evaluate objective function $f(X, r)$.

3. Output: best solution found during enumeration of $\operatorname{Ext}(\mathscr{T})$.

As mentioned before, each point in $\operatorname{Ext}(\mathscr{T})$ is the intersection of three different facets of some cones of the type $N_{i}(u, v)$ and $M_{i}(u, v)$. Hence, the coordinates of each point in $\operatorname{Ext}(\mathscr{T})$ can be computed in constant time. Thus, together with Lemma 10, we obtain the following result.

Theorem 3 For given polyhedral norms $k_{1}, k_{2}$, the enumeration procedure requires $\mathscr{O}\left(n^{4}\right)$ operations to find an optimal solution for Problem (GP).

Example 3 Consider again the problem instance given in Example 1. In order to find an optimal solution, each candidate point $(X, r) \in \operatorname{Ext}(\mathscr{T})$ must be evaluated. Each of these points is obtained at the intersection of three facets of the cones emanating from the fixed points. Thus, for each candidate point we need to solve a simple system of nine linear equations in nine unknowns. To illustrate, consider the solution $(X, r)=((8,8), 8)$ shown in Fig. 4 which is the intersection of selected facets from cones emanating from $A_{3}, A_{4}$, and $A_{5}$. The corresponding system of equations is given by:

$$
\begin{gathered}
\left(A_{3}, 0\right)^{t}+\alpha_{1}^{(1)}(1,-1,1)^{t}+\alpha_{2}^{(1)}(1,0,0)^{t}=(X, r)^{t}, \\
\left(A_{4}, 0\right)^{t}+\alpha_{1}^{(2)}(1,-1,1)^{t}+\alpha_{2}^{(2)}(0,-1,0)^{t}=(X, r)^{t}, \\
\left(A_{5}, 0\right)^{t}+\alpha_{1}^{(3)}(-1,-1,1)^{t}+\alpha_{2}^{(3)}(0,-1,0)^{t}=(X, r)^{t},
\end{gathered}
$$

with unique solution $(X, r)=((8,8), 8), \alpha_{1}^{(1)}=\alpha_{1}^{(2)}=\alpha_{1}^{(3)}=8, \alpha_{2}^{(1)}=2, \alpha_{2}^{(2)}=1$, and $\alpha_{2}^{(3)}=4$. Evaluating all other candidate points $(X, r) \in \operatorname{Ext}(\mathscr{T})$ in this manner, we are able to verify that the above solution is superior to all of them, and hence, is an optimal solution. 
We may also show the following result which provides geometric properties of the optimal solutions from the FDS.

Lemma 11 Let $(X, r) \in \operatorname{Ext}(\mathscr{T})$ be an optimal solution for $(G P)$. Then, for the corresponding circle $C(X, r)$ there exist at least $\mu$ fixed points $A_{i_{k}}$ such that $A_{i_{k}} \in C(X, r), k=$ $1, \ldots, \mu$. We have $0 \leq \mu \leq 3$ and at least $3-\mu$ corner points of $C(X, r)$ lie on the two-dimensional grid formed by all direction lines of norm $k_{2}$ through the fixed points $A_{i}, i=1, \ldots, n$.

Note that for each of the $(3-\mu)$ corner points of $C(X, r)$ identified in the lemma above, the associated fixed point $A_{i}$ must be external to $C(X, r)$ (see Lemma 2).

\section{Conclusion}

We have studied the problem of approximating a point set by the scaled unit circle of an arbitrary norm $k_{1}$, where distance between the scaled unit circle and the point set is measured w.r.t. another norm $k_{2}$. In Lemma 2 we have shown that this distance is a convex and concave function on certain regions, respectively. Furthermore, we have proved some properties of an optimal solution and demonstrated some negative results that differentiate the problem from the Euclidean case. As a special case, we have studied the problem where $k_{1}$ and $k_{2}$ are both polyhedral norms. For this case we were able to derive a finite dominating set containing at least one optimal solution.

Possible lines of further research include the generalization of our problem to higher dimensions. In particular it seems to be possible to transfer results for the polyhedral norm case to higher dimensions. However, the planar version of our problem where $k_{1}$ and $k_{2}$ are smooth or round norms is still not solved. Here, specialized methods, like a geometric branch and bound combined with lower bounds obtained by d.c. decomposition, seem to be promising. A similar approach was successfully applied for the Euclidean version $k_{1}=k_{2}=\ell_{2}$, see [24]. Besides further generalization of our problem also specialization might be interesting. For example, the case $k_{1}=\ell_{\infty}$ and $k_{2}=\ell_{1}$ is interesting since it has many applications in the field of position sensing of objects. Also note that without loss of generality all results for the $k_{1}=\ell_{\infty}, k_{2}=\ell_{1}$ case are also valid for the problem of approximating a point set by an arbitrary rectangle with fixed aspect ratio. Currently, this special case is under investigation and more efficient algorithms are under development.

Open Access This article is distributed under the terms of the Creative Commons Attribution Noncommercial License which permits any noncommercial use, distribution, and reproduction in any medium, provided the original author(s) and source are credited.

\section{References}

1. Brimberg, J., Juel, H., Schöbel, A.: Locating a circle on a sphere. Oper. Res. 55, 782-791 (2007)

2. Brimberg, J., Juel, H., Körner, M., Schöbel, A.: Locating a general minisum circle on the plane. Preprint series of the Institute for Numerical and Applied Mathematics, University of Göttingen, vol. 10 (2009)

3. Brimberg, J., Juel, H., Schöbel, A.: Locating a circle on the plane using the minimax criterion. Stud. Locat. Anal. (17), 45-60 (2009)

4. Brimberg, J., Juel, H., Schöbel, A.: Locating a minisum circle in the plane. Discrete Appl. Math. 157, 901-912 (2009)

5. Brimberg, J., Walker, J.H., Love, R.F.: Estimation of travel distances with the weighted $\ell_{p}$ norm: some empirical results. Journal of Transport Geography, 15, 62-72 (2007)

6. Chan, N.N.: On circular functional relationships. Journal of the Royal Statistical Society. Series B (Methodological), 27, 45-56 (1965) 
7. Chernov, N., Sapirstein, P.N.: Fitting circles to data with correlated noise. Computational Statistics and Data Analysis, 52, 5328-5337 (2008)

8. Drezner, Z.: Facility Location, A Survey of Applications and Methods. Springer, Berlin (1995)

9. Drezner, Z., Steiner, G., Wesolowsky, G.O.: On the circle closest to a set of points. Computers and Operations Research, 29, 637-650 (2002)

10. Drezner, Z., Suzuki, A.: The big triangle small triangle method for the solution of nonconvex facility location problems. Operations Research, 52, 128-135 (2004)

11. Hamacher, H.W., Drezner, Z.: Facility Location: Applications and Theory. Springer, Berlin (2001)

12. Karimäki, V.: Effective circle fitting for particle trajectories. Nucl. Instrum. Methods Phys. Res. A Accel. Spectrom. Detec. Assoc. Equip. 305, 187-192 (1991)

13. Körner, M., Brimberg, J., Juel, H., Schöbel, A.: General minisum circle location. In: Proceedings of the 21th Canadian Conference on Computational Geometry, pp. 111-114 (2009)

14. Labbé, M., Laporte, G., Rodriguez Martin, I., Gonzalez, J.J.S.: Locating median cycles in networks. Eur. J. Oper. Res. 160, 457-470 (2005)

15. Love, R.F., Morris, J.G., Wesolowsky, G.O.: Facilities Location-Models \& Methods. North-Holland, New York (1988)

16. Nievergelt, Y.: A finite algorithm to fit geometrically all midrange lines, circles, planes, spheres, hyperplanes, and hyperspheres. Numerische Mathematik 91, 257-303 (2002)

17. Nievergelt, Y.: Median spheres: theory, algorithms, applications. Numerische Mathematik 114, 573-606 (2010)

18. Pearce, C.E.M.: Locating concentric ring roads in a city. Transp. Sci. 8, 142-168 (1974)

19. Plastria, F., Carrizosa, E.: Gauge distances and median hyperplanes. J. Optim. Theor. Appl. 110, 173-182 (2001)

20. Plastria, F.: GBSSS: the generalized big square small square method for planar single-facility location. Eur. J. Oper. Res. 62, 163-174 (1992)

21. Scholz, D.: Geometric branch \& bound methods in global optimization: theory and applications to facility location problems. Dissertation, University of Göttingen (2010)

22. Scholz, D., Schöbel, A.: The theoretical and empirical rate of convergence for geometric branch-andbound methods. J. Glob. Optim. (2010) doi:10.1007/s10898-009-9502-3

23. Schöbel, A.: Locating Lines and Hyperplanes. Kluwer, Dordrecht (1999)

24. Schöbel, A., Scholz, D.: The big cube small cube solution method for multidimensional facility location problems. Comput. Oper. Res. 37, 115-122 (2010)

25. Suzuki, T.: Optimal location of orbital routes in a circular city. ISOLDE X, Sevilla and Islantilla, Spain, June 2-8 (2005)

26. Ward, J.E., Wendell, R.E.: A new norm for measuring distance which yields linear location problems. Oper. Res. 28, 836-844 (1980)

27. Ward, J.E., Wendell, R.E., Richard, E.: Using Block norms for location modeling. Oper. Res. 33, 1074-1090 (1985)

28. Wesolowsky, G.O.: Location of the median line for weighted points. Environ. Plan. A, 7, 163-170 (1975)

29. Witzgall, C.: Optimal location of a central facility: mathematical models and concepts. National Bureau of Standards Report 8388. Gaithersburg, Maryland (1964) 